\title{
La igualdad y la equidad de género en la educación secundaria costarricense: criterios para un diseño de evaluación
}

\begin{abstract}
Gender equality and equity in Costa Rican education challenges to evaluate
\end{abstract}

\section{Yensi Vargas-Sandoval}

\section{Citar este documento según modelo APA}

Vargas-Sandoval, Yensi. (2021). La igualdad y la equidad de género en la educación secundaria costarricense: criterios para un diseño de evaluación. Revista Actualidades Investigativas en Educación, 21(3), 1-24. Doi. 10.15517/aie.v21i3.48154 


\title{
La igualdad y la equidad de género en la educación secundaria costarricense: criterios para un diseño de evaluación
}

\author{
Gender equality and equity in Costa Rican education challenges to evaluate
}

\section{Yensi Vargas-Sandoval ${ }^{1}$}

\begin{abstract}
Resumen: Este ensayo plantea la discusión sobre la igualdad y la equidad de género en el sistema educativo de secundaria en Costa Rica. Ofrece una propuesta de criterios evaluativos por aplicar con respecto de las acciones de la Política de Equidad e Igualdad de Género promulgada en el Ministerio de Educación Pública. Se realiza una aproximación al contexto social y educativo para plantear el siguiente cuestionamiento general: ¿De qué manera puede contribuir la evaluación social de procesos educativos a develar, en los espacios, las normas y los discursos situados en la convivencia educativa, el binomio igualdad-desigualdad, así como la naturalización e invisibilización de condiciones desiguales desde el género? Desde la disciplina evaluativa, el ejercicio de reflexión se ampara en la perspectiva de evaluación pluralista, que resulta especialmente sensible a las desigualdades, discriminaciones, estereotipos y expresiones violentas relacionados con la condición de género. Este documento ofrece un diseño evaluativo que se elabora luego de la investigación documental y realiza una desagregación metodológica con preguntas, dimensiones, variables e indicadores de carácter evaluativo. Las conclusiones señalan que la evaluación puede y debe contribuir a potenciar ambientes de sana convivencia, libres de discriminación y violencias de género; además, destaca que la temática general de equidad e igualdad en el sistema educativo es responsabilidad de muchas personas y quedan todavía retos por enfrentar cotidianamente, pues todas las personas nos involucramos de distinta forma a los cambios que se requieren para eliminar la discriminación y la violencia por condición de género.
\end{abstract}

Palabras clave: igualdad de género, equidad de género, evaluación educativa.

Abstract: This essay raises the discussion on gender equality and equity in the highschool education system in Costa Rica. It offers a proposal of evaluative criteria to be applied to the actions of the Gender Equity and Equality Public Policy promulgated by the Ministry of Public Education. Through an approach to the social and educational context a general question is posed: In what way can the social evaluation of educational processes contribute to unveiling, in the spaces, the norms and the discourses located in the educational coexistence, the equalityinequality, as well as the naturalization and invisibility of unequal conditions from gender? From the evaluative discipline, the reflection exercise is underpinned by the pluralistic evaluation perspective, which is especially sensitive to inequalities, discrimination, stereotypes and violent expressions related to gender. This document offers an evaluative design that is based upon documentary research and performs a methodological disaggregation with questions, dimensions, variables and indicators of an evaluative nature. The conclusions indicate that the evaluation can and should contribute to promoting environments of healthy coexistence, free of discrimination and gender violence. In addition, it highlights that the general issue of equity and equality in the educational system is the responsibility of many actors and there are still challenges to face on a daily basis, since all of us must be involved in one way or another in the transformations that are required to eradicate gender violence

Key words: gender equality, gender equity, educational evaluation.

\footnotetext{
${ }^{1}$ Investigadora del Instituto de Investigación en Educación, Universidad de Costa Rica, San José, Costa Rica. Orcid https://orcid.org//0000-0002-1644-2247
}

Dirección electrónica: yensi.vargas@ucr.ac.cr

Ensayo recibido: 1 de junio, 2021

Enviado a corrección:30 de junio, 2021

Aprobado: 16 de agosto, 2021 


\section{Introducción}

En el 2017, el Instituto de Investigación en Educación de la Universidad de Costa Rica, finalizó un proyecto de investigación evaluativa en la educación secundaria costarricense, alrededor, principalmente, de las categorías de equidad y de permanencia. En la categoría de equidad, se destacó que lo relacionado con la construcción de género no ha sido suficientemente problematizado y menos si se articula a un enfoque evaluativo.

Al plantear un enfoque evaluativo en un tema tan amplio como la equidad de género, vale la pena retomar que

(...) la evaluación no puede limitarse a documentar y validar condiciones, procesos y resultados educativos con miras estrechas. Ha de analizar, valorar y dar cuenta de los referentes ideológicos, sociales, económicos y políticos que influyen y generan o dificultan ciertas condiciones, prioridades, acciones y resultados en los sistemas escolares. (Escudero, 2010, p. 10)

La Organización de las Naciones Unidas para la Educación, la Ciencia y la Cultura (Unesco) ha aportado, con la Declaración de Incheon y Marco de Acción para la realización del Objetivo de Desarrollo Sostenible 4, la necesidad de definir estrategias para la igualdad de género. Para ello, incorpora no solo el acceso y cobertura de niñas y jóvenes en diversidad de contextos, sino también un llamado para establecer acciones para eliminar discriminaciones e inequidad por razón de género. Se demanda a que "se tomen medidas expresas destinadas a acabar con los prejuicios basados en el género y la discriminación, que se derivan de actitudes y prácticas sociales y culturales y de la situación económica" (Unesco, 2016, p. 32).

En este ensayo se recoge el proceso analítico del contexto social especialmente, entre los años 2018-2020, la referencialidad teórica y la pertinencia de disciplina evaluativa para discutir críticamente la igualdad y la equidad de género en el sistema educativo de secundaria en Costa Rica. Para este propósito, se consideró la Política de Equidad e Igualdad de Género del MEP, en Costa Rica, como instrumental importante para potenciar los cambios necesarios para que los espacios, las normas y los discursos en la convivencia educativa superen las condiciones desiguales desde el género. Resulta meritorio destacar que el diseño evaluativo es un producto concreto del proceso de investigación; sin embargo, la evaluación propiamente dicha aún no se ha puesto en práctica.

El enfoque de investigación cualitativa ha sustentado el proceso, así como la noción de investigación evaluativa, de carácter exploratoria, basada en consulta documental y con 
validación en cuanto a las dimensiones variables e indicadores para la evaluación. Se sustenta la perspectiva de evaluación pluralista como válida para el ejercicio de plantear preguntas de carácter evaluativo, que se desagregan en distintas variables e indicadores.

El diseño evaluativo se sustenta en referentes documentales encontrados y se realiza un marco contextual para luego plantear dimensiones de análisis, preguntas de evaluación, variables e indicadores que relacionan la valoración de planes y estrategias de acción de la Política. Esta propuesta de diseño evaluativo contó con un espacio de validación con profesoras expertas en el Seminario de Evaluación Educativa desarrollado con el IIPEUNESCO.

\section{Desarrollo del tema}

\subsection{Sobre igualdad y equidad de género}

El género es una categoría analítica que las Ciencias Sociales y la Teoría feminista, en particular, han contribuido a ampliar para visibilizar, comprender, explicar y develar, de manera científica, las distintas relaciones de desigualdad según el género atribuido. En un primer momento, la perspectiva de género en la investigación social potenció el análisis del binomio hombres-mujeres y las relaciones de desigualdad y opresión; por lo tanto, posibilitó ensanchar el amplio espectro en que se refleja la desigualdad como un elemento constitutivo de las relaciones sociales, culturales, económicas y por supuesto educativas.

El género es estructural, en tanto la categoría ha funcionado para evidenciar las desigualdades relacionadas con la condición de ser mujer, ser disidente sexual, tener identidades sexuales no binarias y otras expresiones que se desarrollan en la vida cotidiana vinculadas al campo de género:

(...) el género refiere necesariamente al complejo mundo de lo social. Su uso destaca la interacción de un amplio espectro de instituciones económicas, sociales, políticas y religiosas como medio para la producción de los códigos culturales que guían el comportamiento de los hombres y de las mujeres. (Araya, 2003, p. 42)

Por lo tanto, el género es una categoría que profundiza en una dimensión fundamental de lo social y que permite un accionar sobre ello. Además, conlleva a pensar sus articulaciones con otras formas de violencia estructural (clase, raza, colonialismo), por ende, el tema de equidad de género en el campo de la educación traspasa la realidad educativa al ser un asunto que recae en múltiples actores e instituciones sociales, entre ellos la familia, lo comunitario, la 
iglesia, medios de comunicación y otros agentes de carácter social, que constantemente están produciendo discursos sobre el género.

En cuanto a los sistemas escolares, investigaciones en el campo educativo han señalado que el común denominador de la escuela mixta se sustenta bajo el principio democrático de igualdad para todas las personas y defiende la educación conjunta para mujeres y hombres como un compromiso básico del sistema educativo. En consecuencia, la provisión de educación debe ser la misma para todas las personas en el ámbito curricular y pedagógico. Este modelo omite reconocer que, en efecto, existen desigualdades entre los hombres y las mujeres, pues el principio del que parte es el de la "homogenización" de la enseñanza. En consecuencia, el género no es una variable relevante para el trabajo escolar (Araya, 2008).

Guevara y García (2010), en relación con el género y la variable escolar, indican lo siguiente:

En el caso de la escuela, el orden de género se expresa en distintas formas de segregación, discriminación, acoso o la falta de estímulo por parte de docentes y compañeros hacia las estudiantes, ellas encuentran más obstáculos para ser reconocidas y viven más prácticas de exclusión mediante el currículo oculto y el formal. (p. 11)

Al respecto, dicho señalamiento merece especial cuidado, pues este ordenamiento desde el género naturaliza comportamientos incluso violentos en los distintos niveles educativos. En años recientes, el llamado de la UNESCO se dirige a que

tanto en los países ricos como en los pobres las disparidades entre los sexos y las desigualdades de género están muy presentes en el proceso de escolaridad. Prácticamente todos los países tienen disparidades y desigualdades por resolver que ejercen gran influencia sobre el tránsito de varones y niñas por el sistema educativo. (Unesco, 2016, p. 25)

En Costa Rica, el tema de equidad de género está siendo abordado desde la máxima autoridad en educación, el Ministerio de Educación Pública, aunque esta discusión no es tan reciente, pues las primeras propuestas de trabajo datan de la década de los años 1980. Sin embargo, y en consonancia con lo que ocurre en otros países de América Latina, no se logran percibir de manera concreta sus avances. Por ejemplo, referente al currículum oficial se han presentado modificaciones y se realizan diferentes propuestas. Sin embargo, el trabajo no se 
ha materializado, al menos, como se ha planteado, puesto que subsisten actitudes sexistas, violencia de género, desigualdades en lo laboral y otras expresiones que manifiestan el trabajo que aún se requiere en este campo.

Hace años atrás, Lagarde (1996) planteó lo siguiente:

Mujeres y sobre todo hombres que manejan rudimentos de la teoría y la superponen a su propia visión lo hacen sin darse cuenta de que se trata de concepciones antagónicas. [...]. Es posible también que quienes trabajan con la perspectiva de género escindan su conciencia y usen una perspectiva para planificar, presentar proyectos o redactar informes, y no lo utilicen en la dimensión práctica de la organización social, de la cultura y de la política con las organizaciones, las comunidades y las mujeres para las que trabajan, o en sus vidas personales. (p. 09)

Es interesante anotar que hasta el año 2014 se establecieron oficialmente las primeras políticas educativas sobre equidad de género en el Ministerio de Educación Pública, lo cual constituye un leve reflejo de lo retrasado que se ha quedado el sistema educativo costarricense en validar un tema de gran importancia. Esto sugiere una perspectiva desinteresada en esta temática, más cuando se observa que a lo interno del Ministerio se continua abarcando la equidad en acciones concretas como las becas (en lo económico), o en el ámbito tecnológico de accesibilidad, pero no situadas en las desigualdades de género. Con estos aspectos, se verifica la relevancia de continuar con el análisis desde la evaluación de la equidad en la educación costarricense al incorporar particularmente el análisis de la categoría de género (Vargas, 2018).

Desde la perspectiva de la investigación evaluativa, es menester indicar qué se entiende por género y las nociones asociadas con igualdad y equidad. Al respecto, Unesco (2018), en el Informe de Seguimiento de la Educación en el mundo, señala que se siguen centrando en un concepto amplio de la igualdad de género que va más allá del simple recuento del número de niños y niñas en las aulas (p. 7).

Este informe adiciona que, para efectos de la rendición de cuentas, la forma en que se considera la desigualdad de género incide en la naturaleza, el enfoque y los límites de dicha rendición de cuentas. Puntualiza en que persisten tres nociones básicas: una primera que "examina las posibilidades encaminadas a garantizar la paridad de género en la participación y los resultados del aprendizaje, dejando de lado las cuestiones relacionadas con las relaciones de poder que conducen a la desigualdad de género" (Unesco, 2018, p 38). 
Además, señala que se mantiene una perspectiva más analítica, que apunta a presentar y a cuestionar las relaciones y los procesos vinculados con el ejercicio del poder, que conducen a la desigualdad de género. En el nivel más amplio, se refiere a las aspiraciones normativas relacionadas con la justicia o la igualdad en la sociedad más allá de la educación misma (Unesco, 2018, p 38). Estas tres conceptualizaciones parecen resumir las perspectivas clásicas de la investigación y de la evaluación, que podrían interrelacionarse de la siguiente manera: una primera dirigida a la descripción, cuantificación, comparación con estándares previamente definidos que no discuten el arraigo de las desigualdades solo se sustentan en la descripción. La segunda, más analítica y reflexiva, conduce a establecer relaciones y considerar los ejercicios del poder; ciertamente, supera la mera descripción aunque no incorpora un afán político de cambio. En el tercer nivel, se plantean ya no solo las desigualdades en la educación misma, sino también las apuestas por la transformación social y un cambio social y cultural.

Lograr la igualdad de género representa un elemento crucial en la educación como una tarea conjunta de diversidad de personas involucradas. Así, es necesario evaluar las acciones educativas en procura de dicha igualdad, las cuales no pueden dirigirse aisladamente a una instancia o institución. Requieren una visión integradora de las múltiples instancias, niveles y personas involucradas. En ese sentido, las nociones de igualdad y equidad de género se consideran interrelacionadas e integradas en el marco complejo de las relaciones educativas:

Equidad significa igualdad de oportunidades basada en las capacidades humanas, que tod@s debemos tener la posibilidad de desarrollar mediante la educación, y el acceso a una vida larga y saludable. La Equidad se asocia con la justicia, como base del bien que una sociedad debe garantizar a sus miembros. Para que exista Equidad es necesario que en los aspectos humanos fundamentales y en sus posibilidades de desarrollo nadie esté en desventaja. Por eso la Equidad, no es individual, existe en lo social, y debe guiar la distribución de poder, derechos y opciones. (Carosio, 2017, p. 1)

Dichos propósitos han sido señalados y reiterados en convenciones y tratados internacionales, que muchos países han ratificado. Por ello, es importante desplegar ejercicios de evaluación dirigidos no solo al ambiente de aprendizaje, sino también a las funciones y desempeño de gobiernos y centros educativos concretos, así como con el papel que desempeñan docentes y estudiantes en las relaciones cotidianas que estructuran lo social. 


\subsection{Pertinencia metodológica de la evaluación}

El proceso metodológico está sustentado en la perspectiva de investigación evaluativa. Se articula la rigurosidad de la investigación y el potencial metodológico desde la perspectiva pluralista y su rechazo a la idea de evaluación 'imparcial' y 'objetiva', y se traduce en una propuesta 'sensible' a los intereses de los actores que participan en la evaluación" (Izquierdo, 2008, p. 116). La evaluación pluralista es una opción pertinente en la medida que incorpora la visión de distintas personas involucradas y pone en discusión la perspectiva más clásica de evaluar el cumplimiento de objetivos.

Alejados del rigor científico y la imparcialidad de la evaluación clásica, el modelo alternativo va a centrarse en la importancia de los valores, intereses y opiniones de la pluralidad de actores que intervienen en un programa. En la medida en que la evaluación pluralista legitima la diversidad de intereses presentes, reconoce la multiplicidad de perspectivas que estos intereses implican, promoviendo la participación activa de los diversos grupos que tienen relación con un programa. (Izquierdo, 2008, p. 121)

En cuanto al sustento epistemológico es de corte comprensivo que, si bien recurre a la descripción del fenómeno social investigado con datos e indicadores recopilados, se dirige más bien a la profundización: "El método comprensivo implica comprender que a su vez significa abarcar, unir, captar las relaciones internas y profundas de un todo al penetrar en su intimidad, respetando la originalidad y la indivisibilidad de los fenómenos" (Gurdián, 2007, p. 157).

La propuesta metodológica que se desagrega en dimensiones, variables e indicadores. Obtuvo aporte de observaciones y sugerencias de parte de profesoras y expertas en el Seminario de Evaluación educativa celebrado en el IIPE-UNESCO Buenos Aires, Argentina.

\subsubsection{Los retos sobre equidad de género}

El Instituto Nacional de las Mujeres (INAMU) es la entidad pública que alberga los esfuerzos estatales en temas relacionados con la equidad y la igualdad de género. Por ejemplo, en la consecución de marcos normativos y políticas públicas que resguarden la condición de las mujeres.

Al respecto, Cordero (2017) reseña que

...en 1998 se crea el Instituto Nacional de las Mujeres, heredero del Centro Nacional para el Desarrollo de la Mujer y Familia, que había sido creado en 1975. Presenta planes 
de acción en el campo de la ciudadanía de las mujeres, acciones contra la violencia doméstica, e impulsa oportunidades para mujeres y hombres a partir de diferentes acciones afirmativas" (p. 5). Este Instituto (INAMU) ha señalado que persisten los estereotipos discriminatorios y actitudes sexistas, para las cuales son necesarias estrategias integrales, estableciendo alianzas y diálogos positivos para la adopción de medidas que contribuyan a eliminar las prácticas socioculturales de carácter discriminatorio. (INAMU, 2018, p. 7)

Por su parte, el Ministerio de Educación Pública (MEP) promulgó la Política de Equidad e Igualdad de Género que se fundamenta en la Ley General de la Administración Pública, la Ley de Promoción de la Igualdad Real de la Mujer y el objetivo VI de la Política de Equidad e Igualdad de Género (PIEG 2007-2017) con el fin de garantizar, desde la perspectiva de los derechos humanos, las mismas oportunidades para todas las personas, pues en Costa Rica prevalecen condiciones sociales y culturales que conducen a la discriminación por condición de género.

EI MEP desde la década de los años 1980 inició con la incorporación de temáticas de género en el sistema educativo costarricense, desde proyectos, convenios, políticas educativas, planes estratégicos, comisiones para la incorporación del enfoque de género en el material y los programas de estudio (Becerra y Molina, 2014, p. 38).

En las siguientes décadas se consolidaron proyectos a nivel regional; por ejemplo, "Mujeres, Género y Desarrollo (1991)", "Apoyo al Sistema de Mejoramiento de Calidad de la Educación Costarricense (1992)", "Construcción de una cultura equitativa de género en la Escuela Líder (1998)", entre otros, todos con la finalidad de construir una cultura educativa libre de construcciones patriarcales.

Este trabajo, que se consolidaba con el pasar del tiempo, urgía del respaldo de una política educativa; en 1994, se decretó la Política Educativa Hacia el Siglo XXI y se logró conformar en 1995 la Oficina de la Mujer del MEP, cuya función era el fomento y la promoción de temas relacionados con el género.

Para el año 2000, el MEP en conjunto con el INAMU desarrollan un plan estratégico que terminó por consolidarse en el 2001, que se denominó "EI Sistema Educativo hacia una Cultura de Equidad de Género". Contiene acciones con carácter estratégico que permitirian crear las condiciones para la transversalización del género en materia educativa. En el 2004, se instauró la Comisión de Equidad de Género, donde su principal responsabilidad era la construcción de 
la Política de Equidad de Género del Sistema Educativo Costarricense. En el 2005, se buscaba un Modelo Pedagógico Co-educativo. Se procuró atacar el problema de la invisibilización del papel protagónico de la mujer como se evidenciaba en los programas educativos.

Desde el 2009 hasta el 2012, se lograron obtener, después de una lucha muy larga e intensa, los nuevos programas para la afectividad y la sexualidad integral propuestos como reforma curricular Ética, Estética y Ciudadanía del MEP. No obstante, hasta el año 2013 entró en vigencia.

Entre los años 2012 y 2013, se creó el Programa Escuelas para el Cambio en procura de prácticas de género sensibles en centros educativos de preescolar, primero y segundo ciclos de la educación general básica

En el año 2014, el MEP instauró políticas de igualdad de género entre hombres y mujeres con la finalidad de fortalecer la equidad al sustentarse en la Política para la Igualdad y Equidad de Género (2007-2017). Para ello, se conformó la Comisión Institucional de Género del MEP con la responsabilidad de desarrollar los cuatro ejes de acción en lo que se enfoca: Ministerio de Educación y Vinculación Social, Promoción de igualdad de género y no discriminación, Educación con perspectiva de género, Gestión administrativa con enfoque de género.

Se empezó a trabajar visiblemente el tema de diversidad sexual. Sin embargo, hasta el 2015 con la declaración de una directriz, el MEP logró el espacio libre de discriminación por orientación sexual e identidad de género.

En correspondencia con lo anterior, para acompañar el proceso y la efectiva protección de todas las personas contra cualquier tipo de discriminación, se elaboró un "Manual de Día Nacional contra la Homofobia, Lesbofobia y la Transfobia". Las personas directoras de los centros educativos deben promover el análisis y la aplicación de las recomendaciones al declararse el 17 de mayo como "Día nacional contra la homofobia, lesbofobia y transfobia".

La Unesco señala que un ambiente o clima escolar sin violencia es ideal en las sociedades actuales. De ahí, resulta fundamental considerar la estimación del clima escolar en el espacio educativo como contexto inmediato de las relaciones sociales que elimine los eventos violentos. En el informe se puntualiza que

La violencia relacionada con la escuela es un problema omnipresente en algunos países. Puede ser física, psicológica o sexual; puede tener lugar en la escuela, en el trayecto o en el ciberespacio; y puede incluir intimidación, castigo corporal, maltrato verbal y emocional, intimidación, acoso y agresión sexual, actividad de pandillas y presencia de armas entre los estudiantes. A menudo se perpetra como resultado de normas y 
estereotipos de género y se mantiene a raíz de relaciones de poder desiguales. (Unesco, 2018, p. 53)

En este contexto, se requiere resaltar la necesidad de que los planes de acción desplegados de la Política de Equidad e Igualdad de Género del MEP, que pretenden la igualdad y la equidad de género en el contexto educativo, deben necesariamente considerar las relaciones entre pares y las relaciones con el profesorado, así como un contexto amplio en que también se discuta el espacio, la apropiación de dichos espacios y la configuración identitaria desde la categoría de género.

Este hallazgo importante ${ }^{2}$ de la relación entre la política promulgada por el INAMU y la planteada desde el MEP, derivó en el interés en la elaboración, no solo de variables y parámetros sobre equidad de género, sino también una propuesta que guíe una evaluación desde la perspectiva pluralista y de aprendizaje de todas las personas involucradas, considerando que el resultado de toda evaluación debería acrecentar el conocimiento y que se constituya en un aprendizaje más que una estrategia de control (Niremberg, Brawerman y Ruiz, 2003, p. 91).

En el ejercicio de proponer un diseño evaluativo, relacionado con la Política de equidad de género y su plan de acción en Costa Rica, surgen interrogantes sobre dichos atributos; es decir, cómo transferir dichos atributos a una propuesta de evaluación que abarca complejas relaciones no solo institucionales sino también personales. La complejidad de las situaciones en la equidad y la igualdad de género conducen a plantear que el carácter integral de la evaluación debe considerar el contexto amplio en un ejercicio disciplinar concreto que conduzca a valorar, evaluar, un objeto definido, que, a su vez, produzca efectos para la mejora de la situación evaluada.

Entre los ejes y los temas propuestos en la Política de Equidad e Igualdad de Género del MEP están los siguientes: Cultura institucional libre de violencia y discriminación por condición de género; Lenguaje inclusivo; Políticas de contratación laboral con enfoque de género; Incorporación del principio de corresponsabilidad en el cuido familiar (MEP, 2017). Uno de los ejes, que se refiere a la promoción de la equidad e igualdad entre las personas que

\footnotetext{
2 Esta relación se puede ampliar en el Informe final Vargas, Yensi (2018) Investigación evaluativa sobre la educación pública de secundaria: Los criterios de equidad y permanencia. Instituto de Investigación en Educación. Universidad de Costa Rica.
} 
integran el MEP, se vincula con el atributo multiactoral de la evaluación. En este aspecto, destaca lo aportado por Cardarelli de la evaluación centrada en los centros educativos:

Los centros educativos, entonces, constituyen organizaciones pedagógicas, administrativas y entornos laborales y socioculturales complejos. Por ello al evaluar un centro educativo nos sustentamos en los principios de la evaluación institucional, entendida ésta como aquella en la cual se determina la efectividad y eficiencia de una institución educativa, como centro de enseñanza y aprendizaje, de trabajo, y como prestadora de servicios a la sociedad. $(2018$, p. 8)

En los centros educativos se expresa el contexto social, cultural y político de la sociedad en su conjunto; por ello, una interrogante central se refiere a ¿qué papel cumple la Política de Equidad e Igualdad de Género del MEP-Costa Rica para incidir en contextos educativos con igualdad y equidad de género y de qué manera se hace efectiva en centros educativos concretos?

\subsubsection{Dimensiones sustantivas y preguntas evaluativas}

El señalamiento de interrogantes que guiarán la evaluación, así como la identificación de dimensiones sustantivas, es la base de un modelo evaluativo a partir de lo señalado por Nirenberg, Brawerman y Ruiz, cuando plantean que un modelo:

Constituye una abstracción, una representación que se construye para comprender y explicar una realidad compleja; el modelo no es la realidad, pero facilita su comprensión para poder operar en ella. Nuestra interpretación de "modelo evaluativo" incluye la forma o manera de evaluar diferentes realidades o fenómenos sociales. (Nirenberg, Brawerman y Ruiz, 2003, p. 134)

La aclaración sobre el objeto que por evaluar requiere delimitación según dimensiones evaluativas y su apuesta operacional en variables e indicadores. Kisilevsky (2009) aporta que los estudios evaluativos deben operacionalizar las preguntas que guían sin que se diluya el carácter complejo y múltiple del problema o del objeto a evaluar. Por ello, una acción fundamental es plantear dimensiones sustantivas ${ }^{3}$ para evaluar con las correspondientes

\footnotetext{
${ }^{3}$ Nirenberg, Brawerman y Ruiz señalan que la determinación de las dimensiones de la evaluación es, quizá, su paso conceptual más definitorio. Para determinar adecuadamente las dimensiones, deben utilizarse criterios clasificatorios. Las dimensiones sustantivas hacen referencia a los propósitos medulares de programas proyectos y políticas (2003, p 144-145)
} 
interrogantes que puedan dar cuenta de la complejidad de las políticas, programas, proyectos o actividades a evaluar. Añade que:

(...) las preguntas que se formulen deben reunir una serie de atributos, a saber: a) ser pertinentes con relación al objeto a evaluar, o sea, el programa; b) ser relevantes con relación a las necesidades sociales e institucionales que le dan origen y, por último, c) ser factibles de ser respondidas con los recursos y el tiempo disponible. (p. 84)

En este sentido, el ejercicio que se presenta, a continuación, remite a las relaciones entre dimensiones, subdimensiones, variables e indicadores con las preguntas que orientarán el proceso de evaluar. Para ese efecto, se definen cuatro dimensiones sustantivas:

1. Dimensión contextual

2. Dimensión clima escolar

3. Dimensión pedagógica

4. Dimensión gestión

La primera dimensión contextual sobre la Política de Equidad e Igualdad de Género del MEP-Costa Rica que discuta los aspectos culturales que prevalecen, visibles o no para incidir en la equidad y la igualdad de género en la educación costarricense. En este ámbito contextual, es importante retomar una subdimensión de desigualdad de género que recoja indicadores existentes sobre brechas y desigualdad de oportunidades. Otra subdimensión discursiva que reflexione sobre pronunciamientos de actores sociales sobre la labor del MEP alrededor de la equidad y la igualdad de género.

Esta podrá ser capturada mediante discursos, pronunciamientos, publicaciones que revelen la valoración de la política educativa en el marco social general del país y la labor del sistema educativo para mejorar el contexto social y cultural sobre igualdad y equidad.

La segunda dimensión, que se denomina clima escolar, refiere a la relación con el espacio educativo, el uso y apropiación del espacio físico y los significados atribuidos para indagar la manera en que estas variables dan indicaciones de la igualdad o desigualdad de género.

La tercera dimensión propuesta es pedagógica. Propone dar cuenta de las relaciones profesor (a)- alumno (a) y entre pares, si se presentan elementos de currículo oculto sobre discriminación en razón de género y de las preferencias sexuales. En esta se plantean variables relacionadas con el lenguaje sexista en las relaciones cotidianas. 
La otra dimensión referente a la gestión, en la cual interesa relacionar variables relativas a la gestión institucional-gerencial, que pueda expresar el interés por favorecer la igualdad y la equidad mediante normativa, pronunciamientos y resoluciones específicas.

En este caso, la propuesta de evaluación se sustenta en la premisa que es importante no solo considerar datos, variables y parámetros cuantitativos, sino también las categorías y criterios de corte cualitativos para valorar el alcance de los planes de acción diseñados en torno a la Política del MEP

\subsubsection{Propuesta de preguntas, variables e indicadores}

Para delimitar la propuesta metodológica de una propuesta evaluativa, se expone la relación entre las dimensiones, preguntas que orientan la evaluación, variables e indicadores.

En el caso de la dimensión contextual propuesta por dos subdimensiones: desigualdad según género y los discursos en el contexto social y cultural. Se propone valorar aspectos culturales tanto del contexto general exógeno y de qué forma se visibilizan en el entorno endógeno de centros educativos.

Entre otras preguntas que guían la evaluación de esta dimensión contextual están las siguientes: ¿Cuáles son las características culturales que reproducen brechas y discriminaciones según género? ¿Cuánto se ha logrado en la paridad en órganos directivos? ¿Existe exclusión por condición de embarazo o de maternidad? ¿Cuáles son las condiciones culturales de la institucionalidad pública en que se inscribe el esfuerzo del MEP por la igualdad y la equidad de género? ¿Cómo se enfrenta el embarazo adolescente en el sistema educativo en general y en los centros educativos en particular? ¿Tienen trato igualitario las estudiantes madres y los estudiantes padres? En los procesos para prevención del embarazo a temprana edad, ¿están considerando la igualdad y la equidad de género? Esta relación entre dimensión variables e indicadores se puede apreciar en la tabla 1 se aprecian las dimensiones, variables e indicadores. 
Tabla 1.

Dimensión contextual: variables e indicadores

Dimensión contextual: se propone valorar aspectos culturales (exógenos y endógenos)

\begin{tabular}{|c|c|c|}
\hline Subdimensión & Variables & Indicadores \\
\hline \multirow[t]{2}{*}{$\begin{array}{l}\text { Desigualdad según } \\
\text { género }\end{array}$} & Brechas de género & $\begin{array}{l}\text { Paridad de género, en MEP, en Consejo } \\
\text { Superior de Educación y Direcciones } \\
\text { Regionales del MEP } \\
\text { Salarios que devengan hombres y mujeres } \\
\text { en el MEP } \\
\text { Datos sobre graduación de hombres y } \\
\text { mujeres en la educación secundaria } \\
\text { (estadísticas anuales) } \\
\text { Exclusión según embarazo adolescente }\end{array}$ \\
\hline & $\begin{array}{ll}\text { Discriminación } & \text { por } \\
\text { condición de género }\end{array}$ & $\begin{array}{l}\text { Eventos de discriminación documentados } \\
\text { en la Dirección de Asuntos Estudiantiles } \\
\text { Eventos o actos violentos registrados según } \\
\text { la preferencia sexual } \\
\text { Exclusión según embarazo adolescente }\end{array}$ \\
\hline
\end{tabular}

Fuente: elaboración propia, 2021

Se plantea la relevancia de otra dimensión evaluativa relacionada con el clima escolar para valorar cuánto y cómo se logra una convivencia libre de discriminaciones y actos violentos, que relaciona no solo el espacio físico, sino también el espacio social; es decir, la significación social del espacio educativo.

Dentro de las preguntas que se pueden plantear están las siguientes: ¿Se ofrecen o se dispone de espacios físicos de manera igualitaria? ¿El uso de los espacios es igualitario? ¿El espacio físico está disponible para aprendizajes, la recreación y la convivencia? ¿Aparecen o persisten apropiaciones diferenciadas del espacio escolar según género? ¿Existen expresiones visibles o no visibles sobre el uso del espacio según género o identidad sexual? ¿Se califica el espacio según sea utilizado por género? ¿Se encuentran riesgos o peligros diferenciados por género en los espacios?

Se plantean dos subdimensiones sobre el espacio, una relativa a los físicos y otra relacionada con la construcción social, que se puede apreciar en la tabla 2. 
Tabla 2.

Dimensión clima escolar: variables, indicadores

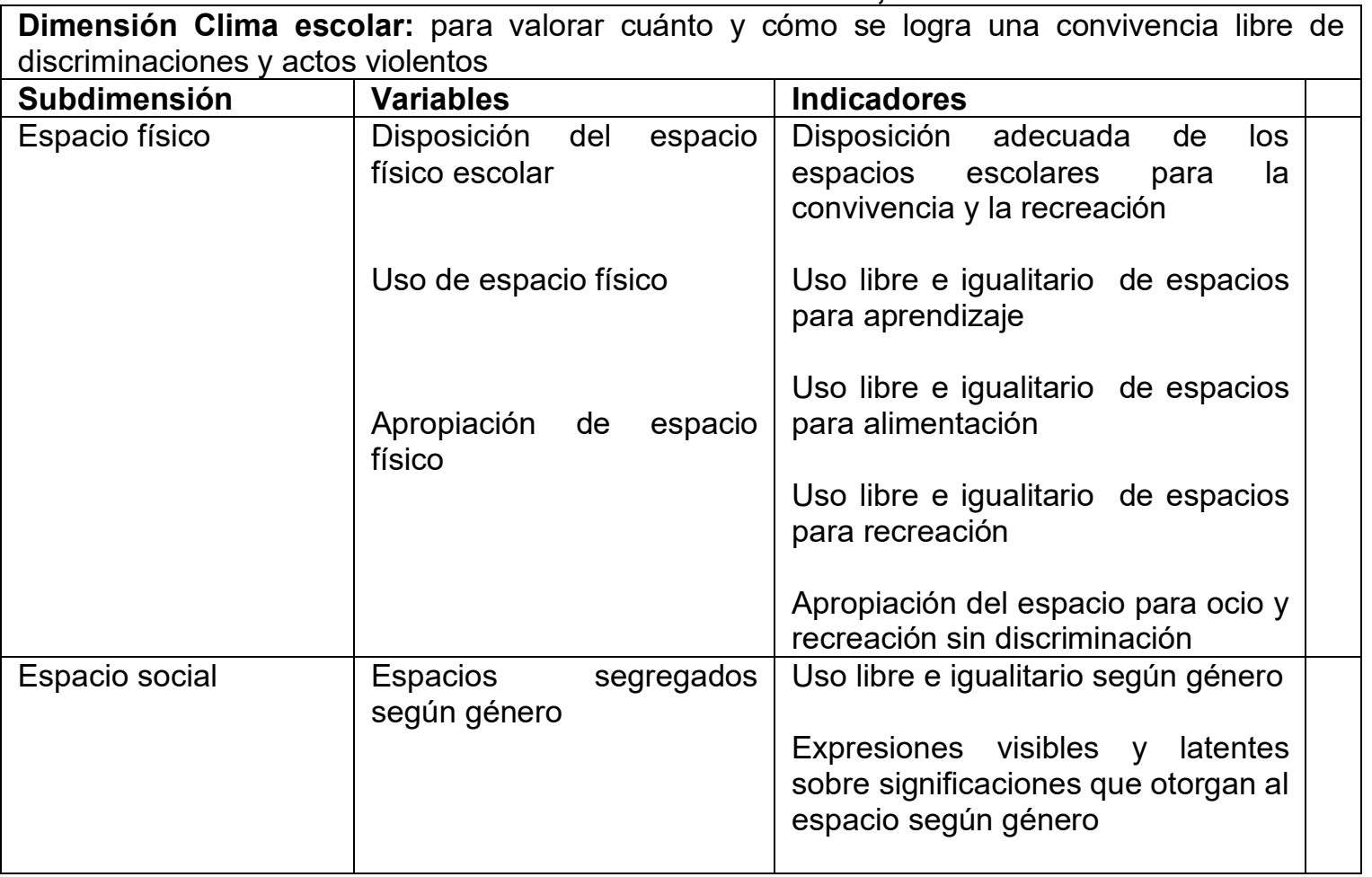

Fuente: elaboración propia, 2021

Otra dimensión que parece apropiada se refiere al ámbito pedagógico relaciona el desarrollo del currículo formal y la prevalencia del currículo oculto en cuanto a discriminaciones y desigualdades según el género. Se suma a la consulta sobre la forma en que se atiende lo relativo a la diversidad sexual y sus repercusiones en el ámbito pedagógico. Dentro de esta, se plantean preguntas orientadas a si, en la relación pedagógica, ¿se presentan eventos discriminatorios? La diversidad de preferencias y vivencias de la sexualidad, ¿se tratan con respeto y en igualdad de condiciones? ¿Se presentan eventos o casos de violencia en los centros educativos que se sustenten por las preferencias sexuales? ¿Se presentan chistes o burlas según las preferencias sexuales? En la tabla 3 se presentan las variables e indicadores que se estimarían en este ámbito pedagógico. 
Tabla 3

Dimensión pedagógica: variables e indicadores

\begin{tabular}{|c|c|c|}
\hline \multicolumn{3}{|c|}{$\begin{array}{l}\text { Dimensión pedagógica relaciona el desarrollo del currículo formal y la prevalencia del currícul } \\
\text { oculto en cuanto a discriminaciones y desigualdades según el género }\end{array}$} \\
\hline Subdimensión & Variables & Indicadores \\
\hline \multirow[t]{2}{*}{$\begin{array}{l}\text { Currículo formal y } \\
\text { oculto }\end{array}$} & $\begin{array}{l}\text { Currículo oculto: presencia de } \\
\text { ciertos tipos de discriminación y/o } \\
\text { violencia } \\
\text { Presencia de eventos de violencia }\end{array}$ & $\begin{array}{l}\text { Lenguaje discriminatorio según } \\
\text { preferencia sexual } \\
\text { Utilización de expresiones verbales } \\
\text { discriminantes por parte de } \\
\text { profesores } \\
\text { Expresiones verbales } \\
\text { discriminantes por parte de alumnos }\end{array}$ \\
\hline & $\begin{array}{l}\text { Respeto por } \\
\text { sexuales diversas }\end{array}$ & $\begin{array}{l}\text { Circulares que indican la igualdad } \\
\text { de trato y el respeto por diversas } \\
\text { expresiones de la sexualidad. } \\
\text { Reporte de casos de violencia en } \\
\text { los centros educativos por la } \\
\text { preferencia sexual. } \\
\text { Chistes y burlas según preferencias } \\
\text { sexuales diversas. } \\
\text { Expresiones de irrespeto por las } \\
\text { preferencias sexuales diversas. }\end{array}$ \\
\hline
\end{tabular}

Fuente: elaboración propia, 2021

Otra de las dimensiones evaluativas que se proponen está relacionada con la gestión de los centros educativos en sus normas y discursos, que pueden dar cuenta de la forma en que el accionar institucional está operando a favor de la eficacia de la política diseñada y favoreciendo ambientes libres de violencia y discriminación según género. En esta, se articulan interrogantes como ¿Cuáles reglamentos internos del MEP se han adecuado según las exigencias de la Política? ¿Cuáles cambios reglamentarios se han propiciado y efectuado? ¿Cuáles acciones concretas se han diseñado para potenciar la igualdad de género? ¿Cuáles aspectos faltan por plantear en los pronunciamientos oficiales del MEP? Se pretende evaluar los aspectos normativos y discursivos. En la tabla 4, a continuación, se resumen las variables e indicadores. 
Tabla 4.

Dimensión Gestión: variables, indicadores

\begin{tabular}{|c|c|c|}
\hline $\begin{array}{l}\text { Dimensión Ge } \\
\text { eficacia de la pc }\end{array}$ & $\begin{array}{l}\text { forma en que el acciona } \\
\text { sus normas y discursos }\end{array}$ & stitucional está operando a favor \\
\hline Subdimensión & Variables & Indicadores \\
\hline Normativa & $\begin{array}{l}\text { Garantía de derechos en } \\
\text { cuanto a igualdad de } \\
\text { género en reglamentos }\end{array}$ & $\begin{array}{l}\text { Reglamento de evaluación } \\
\text { Reglamento de orden y disciplina } \\
\text { Reglamento contra el Hostigamiento } \\
\text { sexual }\end{array}$ \\
\hline Discursiva & $\begin{array}{l}\text { Pronunciamientos orales y } \\
\text { escritos desde el MEP, que } \\
\text { manifiesten o no eliminar la } \\
\text { discriminación. }\end{array}$ & 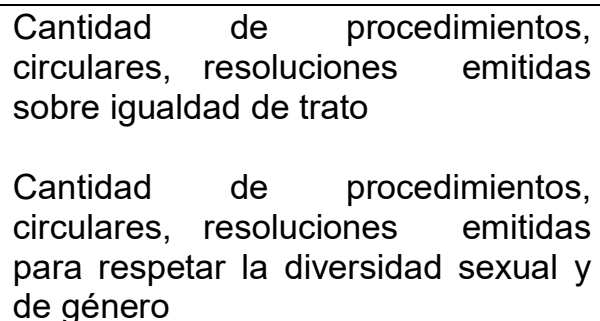 \\
\hline
\end{tabular}

Fuente: elaboración propia, 2021

Los retos son amplios para evaluar, pues se apunta a conjugar dimensiones amplias con variables e indicadores de corte cuantitativo y cualitativo. Sin embargo, la temática de igualdad y equidad de género requiere una amplia discusión y revisión crítica de lo que acontece en los centros educativos y las múltiples maneras en que se incorporan o no en las relaciones sociales-educativas la igualdad de género y el aporte a ambientes escolares de convivencia sana, libre de violencia y discriminación.

\section{A manera de conclusión}

En la perspectiva de la evaluación como aprendizaje, es fundamental tomar en cuenta la importancia de la equidad e igualdad de género en el sistema educativo, en el tanto las personas estamos involucradas para aportar a los cambios necesarios para la eliminación de la discriminación y la violencia amparada al sistema patriarcal y heteronormativo.

Una dimensión potente para indagar y profundizar es la referente a la convivencia escolar que, según nuestra apreciación, consideraría las múltiples intersecciones de la gestión institucional, clima escolar con la apropiación de los espacios y la incidencia de un contexto cultural, que logre una convivencia libre de discriminaciones y de actos violentos.

En el esfuerzo por recopilar parámetros, que permitan valorar la igualdad de equidad de género en la educación, se denota la dificultad por encontrar vinculados los indicadores referidos a igualdad y equidad de género cuyo centro de interés sean las condiciones de desigualdad que se enfrentan en los ámbitos sociales y educativos. 
Difícilmente, se recuperan datos sobre brechas de género, más cuando se registra el mejor desempeño de mujeres en los distintos niveles educativos. Sin embargo, la relación de estos indicadores con la condición de ser madre o de embarazo parece no haber sido profundizada.

Además, en cuanto al personal docente en el ámbito educativo

la feminización del personal docente en la mayoría de los países es un fenómeno bien conocido; en cambio, se presta menos atención al desequilibrio persistente que impera, a favor de los hombres, en los puestos de dirección de la enseñanza y otros cargos directivos conexos. (Unesco, 2018, p. 24)

Por lo tanto, corresponde a una dimensión que merece especial incorporación en otros esfuerzos de evaluación, en que se pueda comparar la participación paritaria en puestos directivos y de representación, la desigualdad de incentivos y de carrera profesional, la brecha salarial, entre otros.

Desde los aportes de la evaluación pluralista que hace "su rechazo a la idea de evaluación 'imparcial' y 'objetiva', este diseño se traduce en una propuesta 'sensible' a los intereses de los actores que participan en la evaluación" (Izquierdo, 2008, p. 116). Esto por cuanto la evaluación pluralista trata de ir más allá de los objetivos y los resultados del proyecto, programa o política.

En el caso de la igualdad y la equidad de género en la educación, un primer nivel de incidencia de resultados de la evaluación será potenciar la incidencia en la definición de políticas desde el Instituto de Investigación de la Universidad de Costa Rica.

Falta mucho por trabajar, evaluar y mejorar. Las tareas quedan planteadas para proporcionarles seguimiento.

\section{Referencias}

Araya, Sandra. (2003). Relaciones sexistas en la educación. Revista Educación, 27(1), 41-52. Recuperado de https://www.redalyc.org/pdf/440/44027105.pdf

Araya, Sandra. (2008). Contenidos y alcances de la equidad de género en las políticas educativas. Recuperado de http://repositorio.inie.ucr.ac.cr/handle/123456789/304

Becerra, Irina. y Molina, Rosario. (2014). Política para la igualdad y equidad de género de la Universidad Nacional, desde la perspectiva docente y estudiantil del curso: Didáctica del Español y los Estudios Sociales, de la carrera Bachillerato y Licenciatura en Pedagogía con énfasis en I y II ciclo, Unidad Académica División Educación Básica, de la Universidad Nacional (Tesis de Licenciatura). Universidad Nacional, Heredia. 
Cardarelli, Graciela. (2018). Abordajes evaluativos para distintos objetos del campo educativo. La evaluación de políticas y programas. Buenos Aires: IIPE-UNESCO.

Carosio, Alba. (2017). Perspectivas feministas para ampliar horizontes del pensamiento crítico latinoamericano. En: M. Sagot (Coord.), Feminismos, Pensamiento Crítico y Propuestas alternativas en América Latina. Argentina: CLACSO.

Cordero, Teresita. (2017). Un breve recuento sobre las acciones para la equidad de género en la Universidad de Costa Rica. Cuadernos Pagu, (49), e174909. Recuperado de https://www.scielo.br/j/cpa/a/XwQjTnkpfnQYnL7fCvZBQBm/abstract/?lang=es\#Modal Downloads

Escudero, Juan. (2010). Evaluación de las políticas educativas: cuestiones perennes y retos actuales. Revista Fuentes, 10, 8-31. Recuperado de https://revistascientificas.us.es/index.php/fuentes/article/view/2585

Guevara, Elsa. y García, Alba. (2010). Orden de género y trayectoria escolar en mujeres estudiantes de ciencias exactas y naturales. Investigación y ciencia, 18(46), 10-17. Recuperado de https://www.redalyc.org/pdf/674/67413508003.pdf

Gurdián, Alicia. (2007). El Paradigma Cualitativo en la Investigación Socio-Educativa. San José, Costa Rica: AECI.

Instituto Nacional de las Mujeres [INAMU]. (2018). Política Nacional para la Igualdad Efectiva entre Mujeres y Hombres 2018-2030. San José, Costa Rica: INAMU.

Izquierdo, Beatriz. (2008). De la evaluación clásica a la evaluación Pluralista. Criterios para identificar los distintos tipos de evaluación. EMPIRIA. Revista de Metodología de Ciencias Sociales, (16), 115-134.

Kisilevsky, Marta. (2009). La evaluación de políticas y programas: desafíos para la gestión en educación. En E. Martin y F. Martínez (Coords.), Avances y desafíos en la evaluación educativa. Madrid, España: OEI.

Lagarde, Marcela. (1996). Género y feminismo: desarrollo humano y democracia. Madrid, España: Horas y Horas.

Ministerio de Educación Pública [MEP]. (2017). Política de Equidad e Igualdad de Género y su Plan de acción. San José, Costa Rica: MEP. Recuperado de http://repositorio.ciem.ucr.ac.cr/bitstream/123456789/238/1/RCIEM215.pdf

Nirenberg, Olga., Brawerman, Josette. y Ruiz, Victoria. (2003). Programación y evaluación de proyectos sociales. Aportes para la racionalidad y la transparencia. Buenos Aires, Argentina: Paidós.

Unesco. (2016). Educación 2030 Declaración de Incheon Hacia una educación inclusiva y equitativa de calidad y un aprendizaje a lo largo de la vida para todos. Recuperado de http://www.Unesco.org/new/fileadmin/MULTIMEDIA/FIELD/Santiago/pdf/ESP-Marcode-Accion-E2030-aprobado.pdf 
Unesco. (2018). Cumplir nuestros compromisos de igual de género en la educación. Recuperado de https://es.Unesco.org/gem-report/2018 gender review

Vargas, Yensi. (2018). Investigación evaluativa sobre la educación pública de secundaria: Los criterios de equidad $y$ permanencia. Recuperado de http://repositorio.inie.ucr.ac.cr/handle/123456789/34 
Revista indizada en

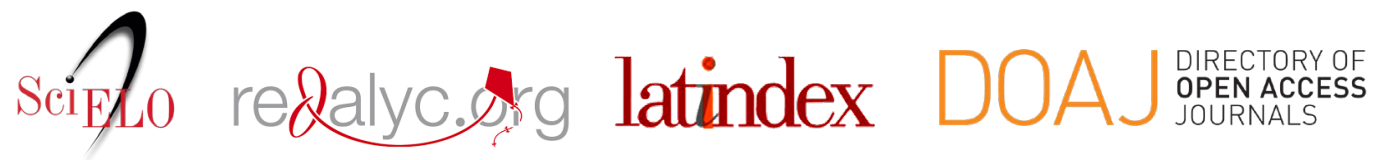

Distribuida en las bases de datos:

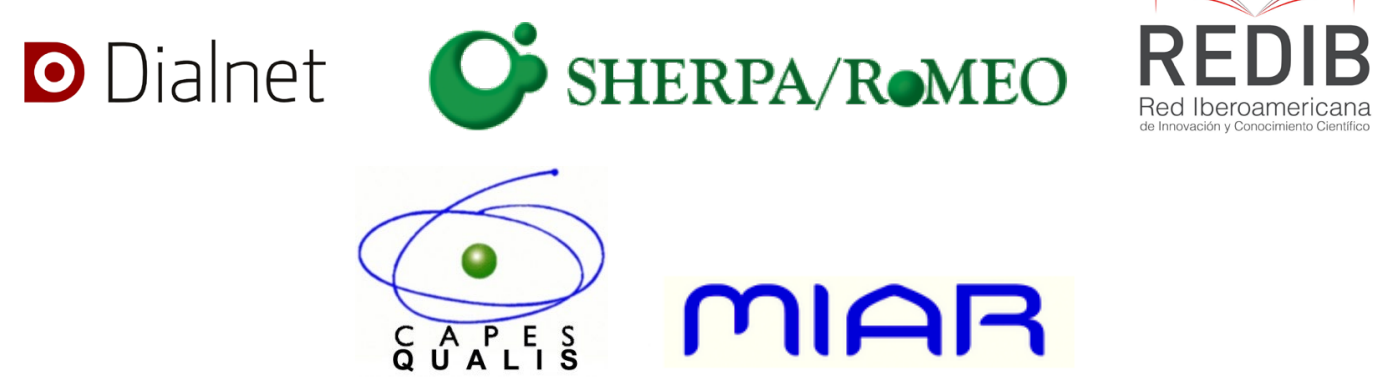

